\title{
Tempoporomandibular joint: Morphological and phisiological aspects
}

\author{
Mauricio Moscovici \\ Instituto de Neurologia Deolindo Couto, Universidade Federal do Rio de Janeiro, Rio de Janeiro, Brazil \\ Email: moscovic@centroin.com.br
}

Received 23 February 2013; revised 15 April 2013; accepted 8 May 2013

Copyright (C 2013 Mauricio Moscovici. This is an open access article distributed under the Creative Commons Attribution License, which permits unrestricted use, distribution, and reproduction in any medium, provided the original work is properly cited.

\begin{abstract}
This is a study where 60 temporomandibular joints were analysed. Method: dissection of anatomical parts, preserving TMJ itself, pterygoid lateral muscle, and its insertions. A special study of the articular disc was performed through histological slicing, for defining its structure and insertions. Results were compared with classical descriptions of different authors. Innervation of related structures is commented, as well as clinical aspects of TMJ conditions in view of its morphological and physiological aspects. Dental occlusion guide alterations, malocclusion, interfere on the TMJ movements, leading to meniscus traumatic compression with possible chronic headache syndrome.
\end{abstract}

Keywords: TMJ Joint; TMJ Meniscus

\section{INTRODUCTION}

Temporomandibular joint dysfunctions present sometimes a set of symptoms that make diagnosis and clinical planning difficult. Usually patients complain of migraine syndrome, severe pains at the TMJ area, with extension towards the hearing apparatus; when moving the mandibula, they say they feel a kind of "click" at the TMJ.

Most clinically observed cases were resultant from dental malocclusion, one or more teeth with supra occlusion (premature contact at closing the mouth), leading to proprioceptive nervous arch modification and small muscular spasms. These muscular spasms may disturb TMJ movements and the meniscus stability. The meniscus then undergoes some traumatic compression as if "bitten" by the condyles bone surfaces.

\section{MAIN REVIEWS AND DISCUSSIONS}

Regarding morphological aspects, TMJ is comparable to any other synovial joint. It presents two joint surfaces- temporal surface and mandibular surface-, an articular capsule, a synovial membrane, and an articular disc. Nevertheless, TMJ presents some morphological and physiological peculiarities (Figures 1 and 2).

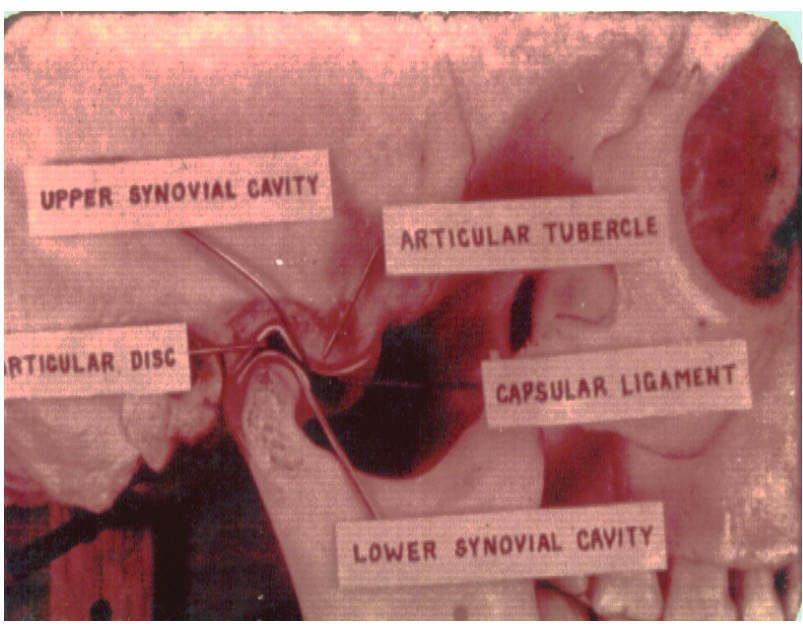

Figure 1. Temporomandibular joint.

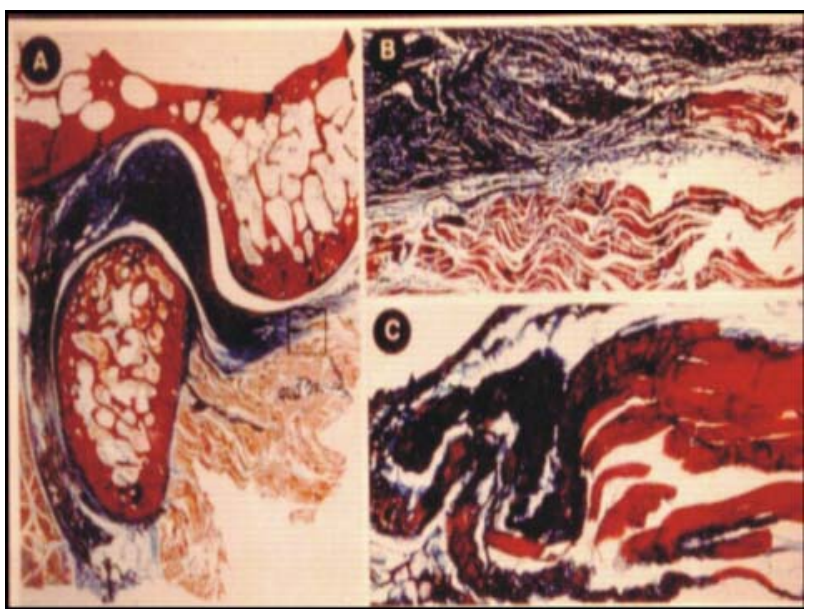

Figure 2. TMJ cross sections. 
TMJ is a bilateral joint, situated between both condyloid processes of the mandible and the skull base. It is not possible to study them on an unilateral basis. Dislocations of one side either move or prevent the movement of the opposite side. The comparison made with the glenohumeral joint by ORTS LLORCA [1], based on fingers interpositioning, is quite illustrative (Figure 3).

TMJ bony surfaces comprehend the articular temporal tubercle and the anterior aspect of mandibular condyle. These are the surfaces that relate in the mandible movements setting. The evidence for this statement is the striking thickness of these surfaces cartilages. Temporal bone mandibular fossa relates to condyloid process in resting state. Mandibular condylloid process directs to mandibular fossa of temporal bone depth, in central occlusion position. Consistent dynamic relationship between articular surfaces is determined by the peculiar morphology of condyloid process, forward and medially twisted. If hypothetical lines traced over condyloid processes lateromedially were medially prolonged, they would meet at the anterior rim of foramen magnum, at skull base (Figure 4).

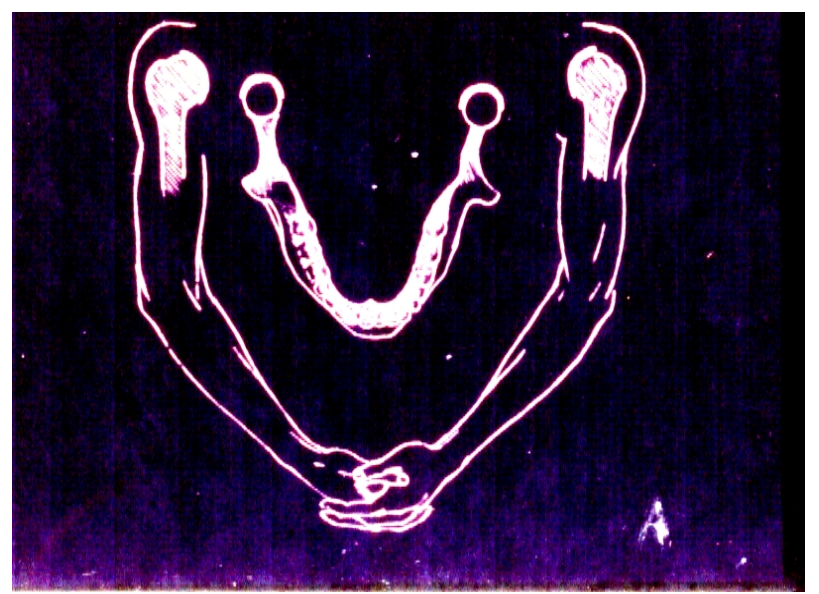

Figure 3. Llorca’s comparison.

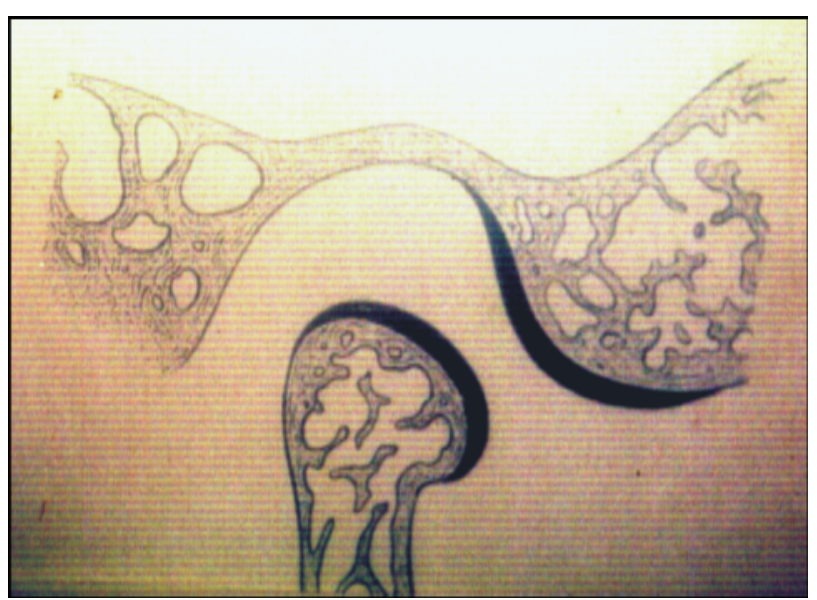

Figure 4. TMJ cartilaginous bone surface.
Muscles actions must also be considered. Superior brain centers exert motor control over mastication muscles, expressed as integrative and harmonious stimulatory and inhibitory actions. There is simultaneous action of muscles from both sides: depressing muscles, lifting muscles, protracting muscles, and retracting muscles. There is either sinergy or antagonism of the muscles from both sides, depending on the movement to be performed. The reflex arch formed by mechanoceptors linked to innervation of mastication muscles that move the mandible, is evident. All these muscles insert themselves in the mandible.

Another important TMJ characteristic is the existence of a guiding line for mandibular movements, corresponding to the occlusal dental interrelation plane. During mastication, mandible moves tracing a trajectory that allows an adequate relationship between both teeth occlusal surfaces. Both form anteroposterior (sagital) and lateral compensatory curves, in such a way that dental cuspids would not intefere during mastication movements. During these mastication movements there is biting and balancing opposite siding. During these movements, condyloid processes dislocate harmoniously along with compensatory curves. These structures trace trajectories which are similar to compensatory curves of teeth occlusal surfaces. A tooth unaligned with the occlusal surface disturbs condyloid processes movements. Anteroposterior condyloid processes dislocations prevail up to the age of 2, when suctioning anteroposterior movements predominate. There are no occlusal compensatory curves at this age, the articular tubercle of the temporal bone is nearly inexistent, and temporal fossa is shallow and sagitally situated. During deciduous dentition TMJ does not present its definitive morphology, due to the fact the teeth do not show compensatory curves yet (Figure 5).

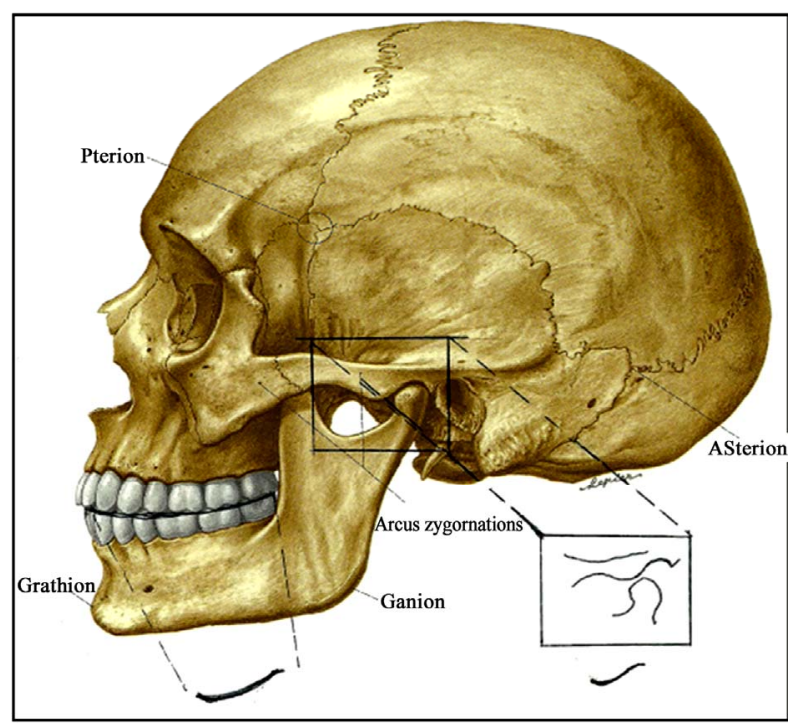

Figure 5. Condyle movement and dental occlusion curve. 
Automatic mandibular movements result from neuromuscular activity. Reflexes originate from mechanoceptors located at TMJ, muscle spindles, and alveolodental ligaments. Proprioceptive fibers of these structures progress up to mesencephalic nucleus of trigeminal nerve. The axons of the latter build synapses with cells of the trigeminal motor nucleus. Correct mastication movements result from homolateral and contralateral neural archs, and are performed through crossed balanced biting, with no interference from teeth cuspids. Mandibular movements are triggered by muscle activity, leading to condyle processes dislocation along with occusal surfaces compensatory curves, under an harmonious trajectory. Condyle processes trace a trajectory consistent with occlusal dental surfaces. The trajectory of the former can be traced through special devices (Figure 6).

There are several classical statements on morphological and physiological aspects of TMJ that ought to be reviewed. CHOUKAS and SICHER [2] refuted many anatomical interpretations regarding this topic, for considering them wrong. The present research corroborates the morphological and physiological conclusions as shared by these authors.

TMJ fibrous capsule surrounds the whole articular complex, inserting itself in the periphery of articular bone surfaces. Fibrous capsule is formed by short and long fibers. Long fibers insert in the mandible and temporal bone. Short fibers insert in each bone surface and in the articular disc, forming a supradiscal cavity and an infradiscal cavity. Both cavities are lined by a synovial membrane, which lubricates the joint.

The existence of external and internal ligaments as a fibrous capsule reinforcement for both joints, as pointed out by several authors, was not confirmed by the current research. Only one thickening at fibrous capsule lateral aspect was found among the 60 joints studied, as it can be considered as a TMJ lateral ligament. No evidence of an articular medial ligament was found. Since it is a bilateral joint situated between mandible and temporal bones, the inexistence of a medial ligament seems physiologically logical to me. Collateral ligaments-medial and lateral ligaments-have important functions in synovial bone joints, as in elbow (between ulna and radius), and knee (between tibia and fibula). Since both TMJ function synergistically as one, both lateral external ligaments work as the collateral ligaments, quoted on the above examples.

Both bundles of the pterygoid lateral muscle dislocate mandibular condyloid processes forward, but not the articular disc, as many authors state. These muscles insert themselves in the neck of the condyle, not in the meniscus (articular disc). Had pterygoid lateral muscle pulled articular disc forward, an antagonist muscle was supposed to pull it backwards. The meniscus insert in condyle process lateral and medial aspects, and it is pulled during the forward and backward movements of the latter. Only upper and more medial pterygoid lateral muscle fibers insert in the anterior rim of the articular disc. The former has a stabilizing function, by preventing articular disc lateral and medial dislocation. Pterygoid lateral muscle, therefore, promotes harmonious movements of the meniscus, along with condyloid process. Corresponding dysfunctions can cause TMJ pathological conditions (Figures 7 and 10).

TMJ articular disc appears to have an anteriorly upper concave and posteriorly convex surface, and a lower concave surface, under a sagital view. Articular disc upper shape would thus be a concave-convex one: condyloid process and mandibula fossa. Inferior concavity of articular disc would represent an adaptation to condyloid process. This description, made by several authors, is nevertheless erroneous. This would be a congruent interpretation for the bony surfaces, but it is inconsistent with the articular dynamics. Articular disc exerts its function

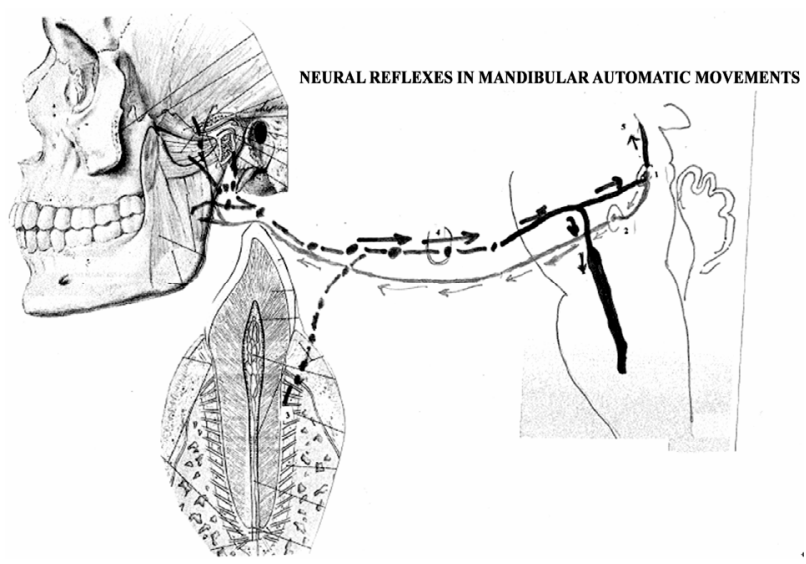

Figure 6. Neural reflexes of mandibular automatic movements.

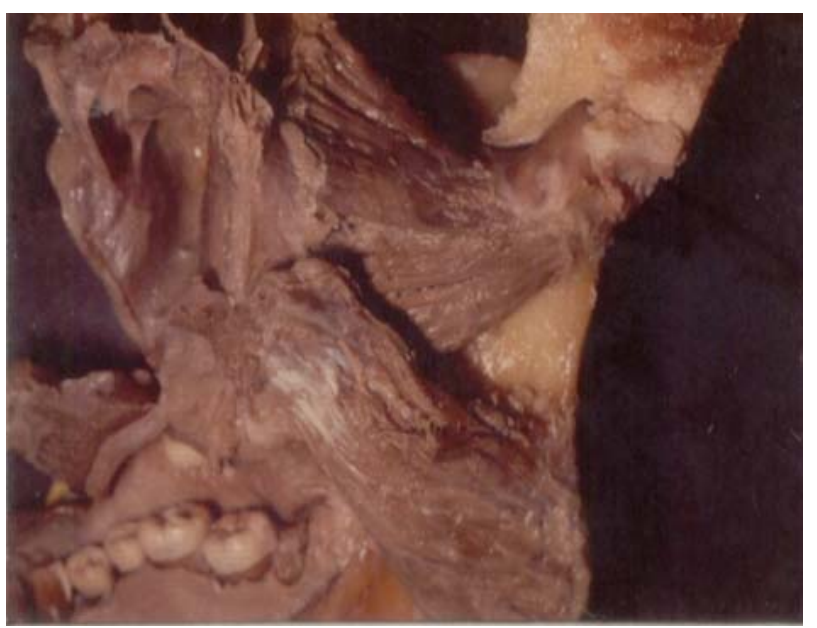

Figure 7. Lateral pterygoid muscle inserted at the neck of the mandibular condyle. 
only between actual articular bony parts: the articular tubercle and the condyloid process, therefore presenting a biconcave shape. The meniscus,articular disc is therefore a biconcave structure,inserted in the lateral and medial poles of the mandibular condyle. Meniscus sets congruence between the temporal articular tubercle and the condyloid process of the mandible, and allows a forward trajectory of mandibular movements. Extension trajectory is determined by two movements: 1 ) a supradiscal movement, which corresponds to a forward movement of mandible condyloid process and the meniscus setting around the articular tubercle of the temporal bone and 2) an infradiscal movement, which corresponds to a rotational movement of the condyloid process inferiorly to the articular disc. Condyle process relates to the articular tubercle through the articular disc, at maximal mouth opening (Figure 8).

The posterior part of the articular disc, macroscopically still considered as articular disc, actually is not meniscus, but a bunch of elastic fibers, that attach the meniscus to the anterior lip of the squamostympanic fissure of the temporal bone and function as meniscus brake, described by SAPPEY [3], as retrodiscal pad. The meniscus is actually a non-irrigated fibro-cartilagenous disc, humidified and nourished through synovial liquid. The retrodiscal pad is composed by elastic fibers, and is intensely irrigated. The meniscus has its collagen fibers arranged in all directions. The retrodiscal pad limits meniscus dislocation, by anchoring its posterior rim to the anterior rim of tympanosquamosal fissure. The elastic fibers of the retrodiscal pad stretch to their limit, when condyloid process - and thus articular disc — is pulled by pterygoid lateral muscle. Part of the fibers of the meniscus pad mingles with the fibrous capsule posterior fibers, inserting itself in the posterior aspect of condyle process neck (Figures 9-13).

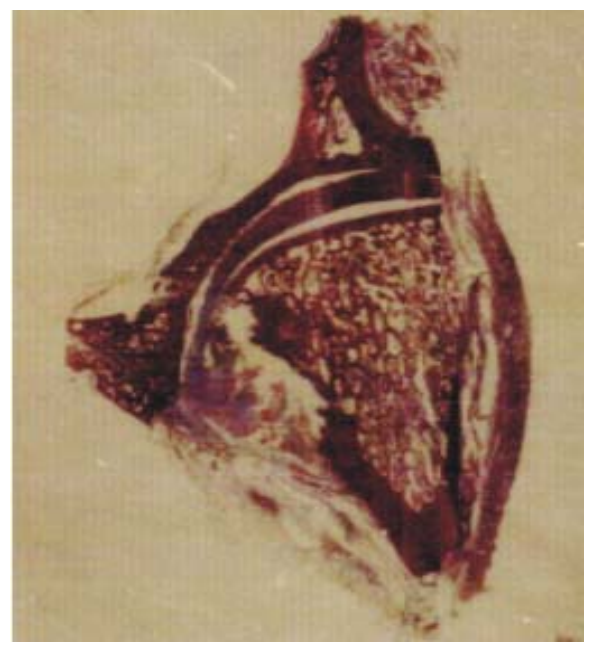

Figure 8. The meniscus inserted in the medial and lateral poles of the mandibular condyle.

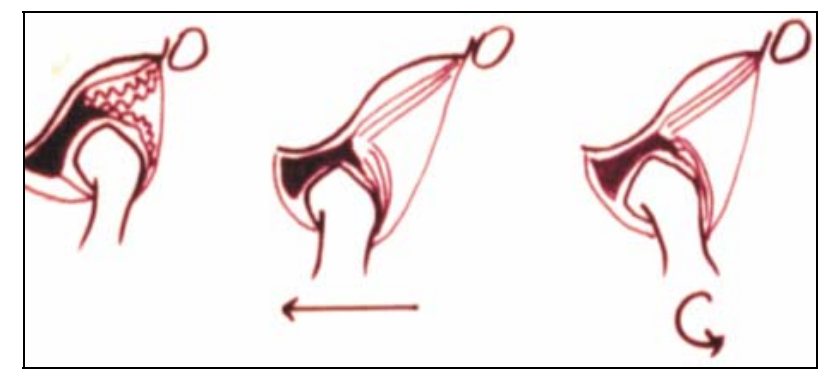

Figure 9. Elastic fibers of the retrodiscal pad and TMJ movements.

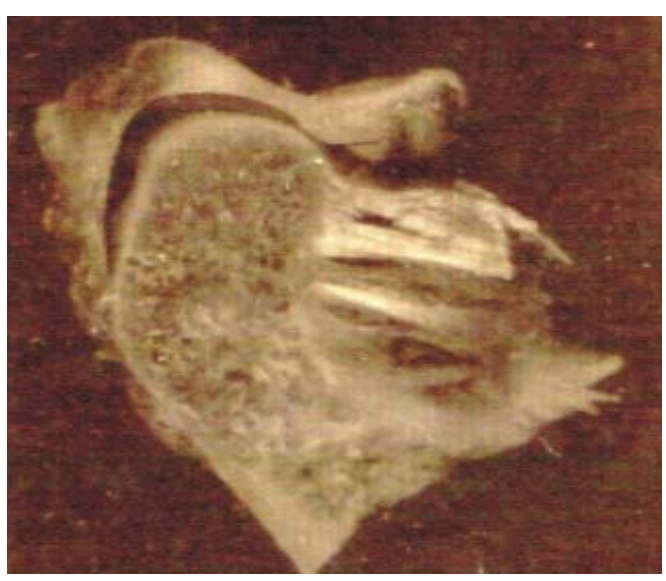

Figure 10. Relations of the articular disc, retrodiscal pad and pterygoid muscle with the mandibular condyle.

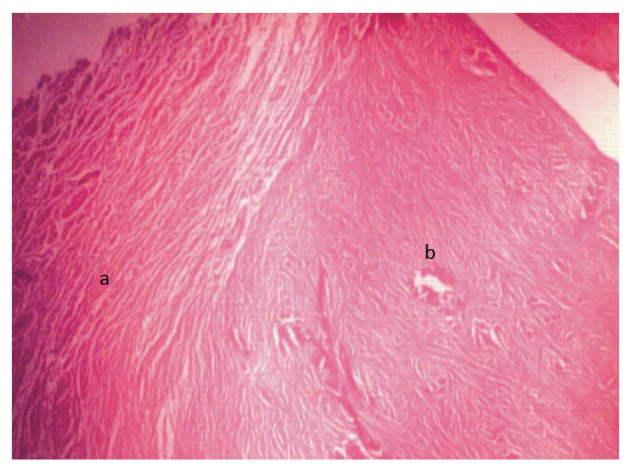

Figure 11. Retrodiscal pad (a) and meniscus (b).

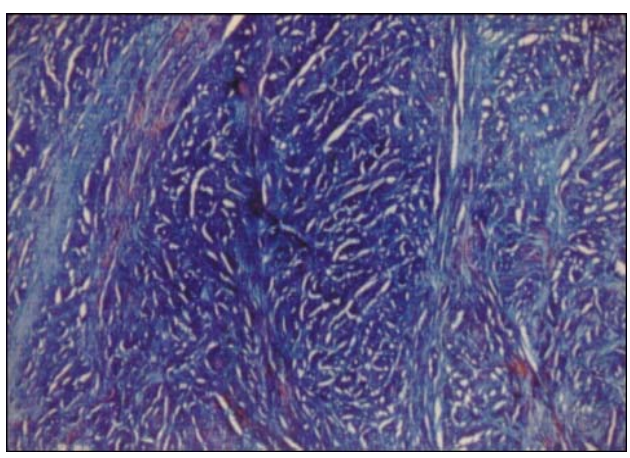

Figure 12. Cross section of the meniscus fibrocartilaginous structure. 


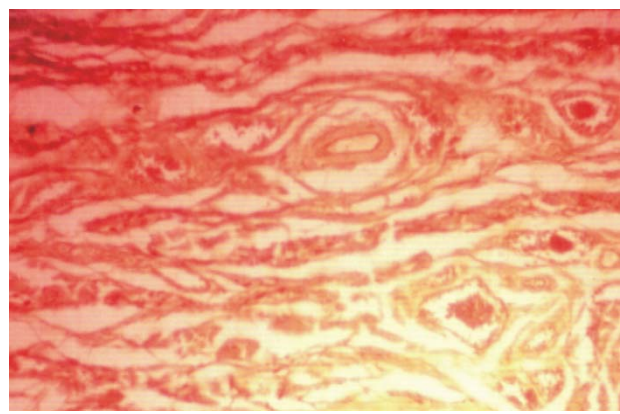

Figure 13. The meniscus elastic retrodiscal pad, richly vascularized.

Mastication movements result from neuromuscular action. Apherent pathways start from proprioceptive receptors located at TMJ, alveolodental ligaments, mastication muscles, tongue, and mouth walls. Malocclusion, presence of a prothesis, or an inadequate dental restoration, can affect mastication movements. Inadequacy from a dental cuspid can affect a balanced biting, compromising TMJ articular disc stability. These irregular movements can cause articular disc trauma, for it is pressed between the articular tubercle and the condyloid process. If there is an early contact of dental surfaces in supraocclusion at mouth closing, then neural arch is compromised. Mouth closing dominant movement at supraocclusion is affected by alveolodental ligament proprioceptive receptors from the early contact region. This condition may cause microspasms of the pterigoid muscles and interfere in the articular disc stabilizing structures. These changes may cause hearing problems, since tensor tympani muscle innervation originates from the nerve to the lateral pterygoid muscle.

Some authors claim having found a ligament linking malleus ossicle from tympanic cavity to TMJ. Notwithstanding, these structures simply represent embryonic remains from the mesenchyma that wrapped Meckel's cartilage and fulfill tympanosquamosal fissure. They neither represent a ligament nor have any explainable function. TMJ inflammatory processes may disseminate from tympanosquamosal fissure to tympanic cavity, and viceversa.

The so-called long distance ligaments-pterygomandibular ligament and stylomandibular ligament-do not have any relationship whatsoever with TMJ. From a phy- logenetic perspective, stylomandibular ligament represents an atrophied muscle found in other animals, with no function in man. Pterygomandibular ligament is a fibrous thickening of the fascia that limits pterygoid lateral muscle and pterygoid medial muscle in-between space, keeps the fatty pad of the cheeks (Bichat's pouch), and shields mandibular vasculo-neural pedicle.

Some authors attribute a variety of symptoms, such as pains and disturbances of hearing, to the pressure upon the auricolotemporal and chorda tympani nerves. This interpretation may not be acceptable, because these nerves do not reach the articular capsule. The auricolotemporal nerve contours the mandibular condyle neck below the capsule. Such a possibility exists in certain cases, when the bone is destroyed and the capsule is ruptured.

\section{CONCLUSIONS}

The study of TMJ should be made together with the dental arch occlusion planes and the mastication muscles. This complex whole constitutes the "mastication apparatus". TMJ physiological principles are similar to any synovial articulation, but its structural elements establish a peculiar dynamics. TMJ should be analyzed bilaterally. Mandibular movements displace both condyles simultaneously. At the rest position, the mandibular condyles stay inside the mandibular fossa of the temporal bone.

Some affirmations on the TMJ morphology and functionality have been repeated for decades without any sound evidence. Other researches are advisable in order to develop better knowledge and effective clinical orientation.

\section{REFERENCES}

[1] Llorca, F.O. (1948) Anatomia humana. Editional Cientifico Médica. Barcelona.

[2] Choukas, N.C. and Sicher, H. (1960) The structure of the temporomandibular joint. Oral Surgery, Oral Medicine, Oral Pathology, 13, 1203-1213. doi:10.1016/0030-4220(60)90093-1

[3] Sappey, M.P.C. (1872) Traité D’Anatomie descritive. A. Delahaye, Paris. 